\title{
Hidatidosis humana en el Perú
}

\author{
Human hydatid disease in Perú
}

\author{
Luis Guerra Montero' \\ Hospital Ramiro Prialé Prialé, ESSALUD
}

\author{
María del Carmen Ramírez Breña ${ }^{2}$ \\ Universidad Continental \\ 18mcrb@gmail.com
}

\section{RESUMEN}

El objetivo de este trabajo ha sido conocer el estado de la hidatidosis humana, por la gran incidencia que presenta en nuestro país, principalmente en la región central andina. La recopilación de datos fue a partir de la revisión de fuentes primarias sobre epidemiología, el ciclo vital del parásito y del manejo del paciente afecto de Hidatidosis. Algunos hallazgos permiten afirmar que la hidatidosis es una enfermedad producida por el estado larval del cestodo Echinococcus del género Granulosus, de alta prevalencia en nuestro país y más en la región central andina, esto se debe principalmente a la costumbre de convivir con perros y ganado ovino así como también a la falta de educación y concientización de la población. Los estudios serológicos como Elisa lgG y Wester Blot por su mayor sensibilidad y especificidad son las pruebas de primera línea para confirmar el diagnóstico. Dependiendo del tamaño, las características del quiste hidatídico y su localización se decide el tratamiento. La hidatidosis es una enfermedad producida por el cestodo Echinococcus Granulosus de alta prevalencia en el Perú y más aún en la región central andina (Cerro de Pasco, Junín, Huancavelica), tiene un lento crecimiento dentro de los órganos humanos pudiendo ser asintomáticos hasta la edad adulta, los órganos más afectados son el hígado y el pulmón donde son diagnosticados por sus antecedentes epidemiológicos, clínica sugestiva (dolor abdominal, vómica) estudios de imagen principalmente ecografía abdominal, radiografía de tórax y exámenes de laboratorio como el Elisa lgG y Wester Blot.

Palabras clave: Epidiomología, hidatidosis hepática, hidatidosis pulmonar.

\section{ABSTRACT}

The objective was to determine the human hydatidosis state, because of the high incidence that occurs in our country, mainly in the central Andean region. Data collection was based on the primary sources review about epidemiology, the parasite life cycle and the treatment of the patient affected by hydatidosis. Some findings support that hydatidosis is a disease caused by the larval stage of the tapeworm Echinococcus of the Granulosus gender, with high prevalence in our country and in the central Andean region, this is mainly due to the habit of living with dogs and sheep as well as the lack of education and population awareness. The serological studies as Elisa IgG and Wester Blot for its higher sensitivity and specificity are the first line tests to confirm the diagnosis. Depending on the hydatid cyst size, characteristics and location, treatment is decided. The hydatidosis is a disease caused by the Echinococcus Granulosus tapeworm of high prevalence in Peru and even more in the central Andean region (Cerro de Pasco, Junin, Huancavelica), it has a slow growth in human organs, they can be asymptomatic until the adult age, the most affected organs are the liver and lung where they are diagnosed because of their epidemiological background, suggestive symptoms (abdominal pain, vomit), imaging studies mainly abdominal ultrasound, chest X-ray and laboratory tests such as Elisa IgG and Wester Blot.

Keywords: Epidemiology, hepatic hydatidosis, pulmonary hydatidosis.

Historial del artículo:

Recibido: 27 de enero de 2015. Aprobado: 12 de abril de 2015. Disponible en línea: 30 de junio de 2015

1 Doctor en Medicina, Especialista en Gastroenterología por la Universidad de la Republica UDELAR Montevideo Uruguay, Medico Asistente de la Unidad de Gastroenterología y del Servicio de Medicina Especializada del Hospital Ramiro Prialé Prialé, ESSALUD, Huancayo.

2 Estudiante de la Facultad de Medicina Humana de la Universidad Continental, Huancayo, Perú. Practicante externa del Servicio de Medici-

na Especializada del Hospital Nacional Departamental de Huancavelica. 


\section{INTRODUCCIÓN}

La hidatidosis es una zoonosis parasitaria, producida por el estado larval del cestodo Echinococcus del genero Granulosus, se caracteriza por la presencia de un quiste a nivel hepático, pulmonar o en ambas localizaciones, además de otras localizaciones no habituales; presentando una alta prevalencia en nuestro país y más en la región central andina, esto se debe principalmente a la costumbre de convivir con perros y ganado ovino así como también a la falta de educación y concientización de la población.

La importancia de esta zoonosis en la salud pública está relacionada no solo con el elevado índice de mortalidad humana, sino también con las pérdidas por rendimiento laboral, gastos de hospitalización, intervenciones e incapacidades.

\section{Epidemiologia}

La hidatidosis es una zoonosis de distribución mundial, conocida desde la época de Hipócrates aparece en toda Europa, especialmente en la región mediterránea y zonas de América Latina, Asia, África y Australia donde puede llegar a tener una incidencia de 50 casos anuales por cada 100000 habitantes (1).

En América Latina los países más afectados son Uruguay, Argentina, Brasil, Perú, Bolivia y Chile donde representa un serio problema de salud pública $(2,3) \mathrm{El}$ Perú en la actualidad, es probablemente el país de las Américas con una mayor incidencia y prevalencia de equinococosis quística, entre el 2001 y 2006 el número de casos por año fue entre 7 y 11 casos por cada 100 000 personas, sin embargo hay departamentos con más altas tasas de incidencias entre 14 y 34 casos por cada 100000 habitantes como es el caso de Junín, Cerro de Paco y Huancavelica $(4,5,6)$, en estos departamentos las provincias de Chupaca, Chaupimarca y Acoria respectivamente son los lugares de donde provienen la mayoría de los pacientes (6).

Aunque en la mayoría de la literatura extranjera se describe que la localización más frecuente de la hidatidosis es la hepática (7-9) en los estudios realizados en el Perú fue pulmonar hasta en un $60 \%$ de los casos $(6,10)$ inicialmente se afirmó que esta inversión de localización podría deberse a que antes del 2000 en los hospitales peruanos era más fácil la accesibilidad a la radiografía del tórax que a la ecografía abdominal, sin embargo los estudios citados se realizaron en los últimos 10 años cuando la accesibilidad a los estudios de imagen en los hospitales eran por igual.

Otra hipótesis seria que en altitudes elevadas se incrementaría la dilatación y volumen sanguíneo capilar en los pulmones que explicaría el predominio pulmonar en el Perú $(11,12)$. Suponemos que la causa de esta inversión es debido a que los pacientes con hidatidosis pulmonar son diagnosticados más frecuentemente ya que la menor elasticidad de su parénquima provocaría más síntomas.

También es importante destacar que ningún órgano es inmune a la infección y en el Perú se han descrito lesiones peritoneales, esplénicas, renales, óseas, tiroideas, mamarias entre otras localizaciones (13).

Respecto a la preferencia por la localización dentro del hígado es predominantemente en el lóbulo derecho y en los pulmones también el del lado derecho similar a otros estudios extranjeros tanto en adultos como en niños $(14,15)$.

La edad del diagnóstico de la hidatidosis hepática en toda Latinoamérica es muy variable se encuentra entre 5 y 80 años, con una relación mujer/hombre en general más uniforme de $1: 1$. Asimismo la hidatidosis pulmonar tiene una edad de diagnóstico que varía de 6 y 68 años, con un leve predomino del sexo femenino $(6,7,10,16)$.

Si bien es cierto no en todos los estudios se informa sobre la convivencia con animales en los estudios que lo reportan el porcentaje es alto $50 \%$ y hasta $80 \%$ de los casos $(6,10,16)$.

\section{Ciclo evolutivo}

Los huevos de Echinococcus granulosus ingeridos eclosionan en el intestino de los huéspedes intermediarios (herbívoros) o accidentales (hombres) y liberan oncosferas el primer estado larval, los mismos que al atravesar la pared intestinal, pasan al torrente sanguíneo y llegan a varios órganos internos principalmente el hígado y los pulmones donde se convierten en metacestodes o hidátides, que son el estado larval propiamente dicho, las que son uniloculares y contienen liquido en su interior (producto del metabolismos larvario) lentamente irán aumentando de volumen y formando protoescolices, vesiculas proligeras, escolices y ganchitos, los que en conjunto se conocen como "arenilla hidatídica" (17). El metacestode además tendrá una pared conformada por una membrana externa cuticular y una interna germinativa, a su vez el órgano parasitado hará una reacción inflamatoria formando la membrana periquistica o adventicia, la hidátide más la adventicia conforman lo que llamamos quiste hidatídico (figura $\left.N^{\circ} 1,2\right)(2,17)$.

Los perros y otros caninos son los huéspedes definitivos de Echinococcus granulosus se infectan al ingerir vísceras con quistes hidatídicos fértiles conteniendo larvas protoescólices que se invaginan y se adhieren a los intestinos de los perros convirtiéndose en estrobilas o parásitos adultos. (2) Las estrobilas (figura $\mathrm{N}^{\circ} 3$ ) 
aparte de la cabeza o escólex, ventosas y cuello tienen $3 \circ 4$ segmentos rectangulares llamados proglotides el último o gravídico presenta mayor tamaño es de forma ovoidea y contiene un promedio de 586 huevos fértiles que son eliminados en la materia fecal del perro (17).

Los huevos pueden tener una larga supervivencia en condiciones ambientales adecuadas de temperatura pueden quedar adheridos al pelaje y al hocico de los perros $(2,17)$.

Los seres humanos se infestan en forma incidental, por convivencia y contacto con el perro que lleva adheridos en sus pelos huevos del parásito o bien por la ingestión de frutas, verduras o agua contaminadas con las excretas del mencionado animal.

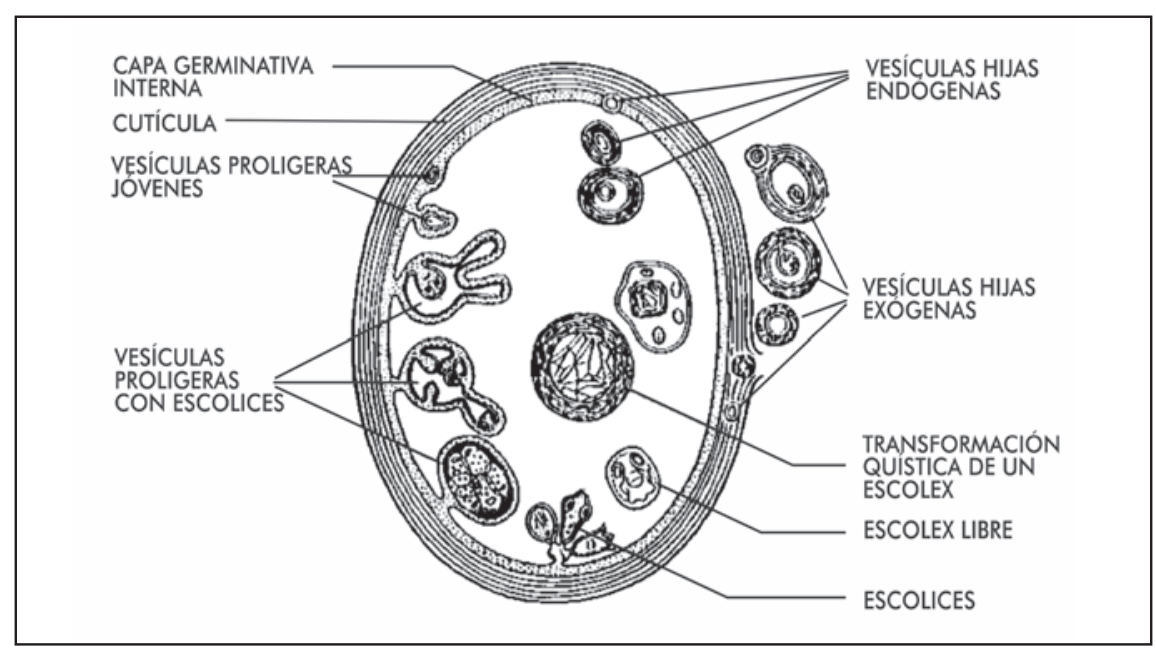

Figura $\mathrm{N}^{\circ}$ 1: Estructura del quiste hidatídico.

Fuente: Electronic Presentation Online System. 2012. Recuperado de: http://posterng.netkey.at/ esr/viewing/index.php? module=viewing_poster\&task=viewsection\&ti $=350164$

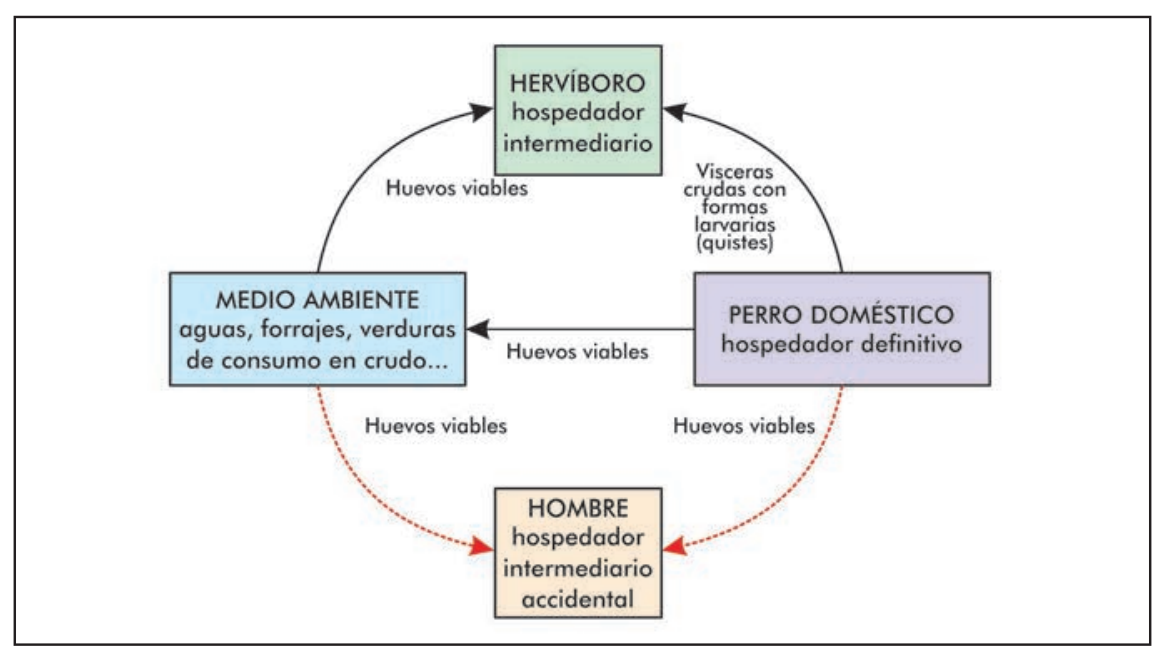

Figura $\mathrm{N}^{\circ}$ 2: Ciclo biológico.

Fuente:MadridSalud. 201 1.Recuperadode: http://www.madridsalud.es/temas/hidatidosis.php.

y humedad pudiendo estar vivas hasta 294 días, depositados en el ambiente con la ayuda del viento, las aves etc. se pueden diseminar hasta una distancia de 170 m y con los dípteros y escarabajos que actúan como transportadores pueden dispersase hasta 30 000 ha contaminando grandes extensiones de áreas de cultivo, pozos de agua y el medioambiente donde deambulan y defecan los perros, así mismo también

\section{Clínica}

Según algunos autores por las características estructurales de mayor resistencia del parénquima hepático el crecimiento de los quistes es más lento o nulo durante años, por lo que un alto porcentaje de personas permanecen asintomáticas durante toda su 
vida o presentan síntomas recién en la edad adulta los mismos que son inespecíficos como epigastralgia, sensación de tumoración abdominal, vómitos, distensión abdominal y con menor frecuencia fiebre, ictericia y ascitis cuando hay apertura del quiste a la vía biliar $(2,17,18)$.

Contrariamente en los pulmones por las características elásticas de su parénquima se ofrece una escasa resistencia al crecimiento del quiste, esto determina un aumento de su tamaño relativamente rápido con la consiguiente aparición de manifestaciones clínicas cuando el quiste alcanza un tamaño de $5 \circ 6 \mathrm{~cm}$. Aunque también las mismas sean inespecíficas como dolor, disnea, tos, al examen físico se pueden encontrar signos de tumoración con matidez, disminución del murmullo vesicular y soplo $(17,10,19)$. que permite establecer el estadio del quiste en base a alteraciones estructurales que caracterizan a los distintos momentos de su evolución para decidir el tipo de tratamiento $(20,21)$.

Así mismo es utilizado para el seguimiento evolutivo de las personas ya tratadas y para guiar la punción si el paciente es tratado con el método de PAIR (Punción, aspiración, inyección y re-aspiración) $(17,22,23)$.

La clasificación ecográfica (tabla $N^{\circ} 1$ ) clásica de Gharbi es la más usada sin embargo la OMS ha propuesto una nueva clasificación con algunas variaciones con respecto a la propuesta por Gharbi de gran importancia terapéutica $(17,22)$.

A pesar que tienen mayor especificidad la indicación de tomografía axial computarizada y resonancia

Tabla $N^{\circ}$ 1: Clasificaciones ecográficas de quiste hidatídico.

\begin{tabular}{|c|c|c|c|}
\hline \multicolumn{2}{|r|}{ Clasificación de Gharbi } & \multicolumn{2}{|r|}{ Clasificación de Gharbi } \\
\hline Tipo & Características ecográficas & Tipo & Características ecográficas \\
\hline & & $\mathrm{CL}$ & $\begin{array}{l}\text { Quiste de características inespecíficas y } \\
\text { contenido liquido homogéneo } \\
\text { Indistinguible de quiste simple }\end{array}$ \\
\hline I & Colección liquida pura & CE 1 & Corresponde al Tipo I de Gharbi \\
\hline ॥ & $\begin{array}{l}\text { Colección liquida con membranas separadas } \\
\text { de la pared }\end{array}$ & CE2 & Corresponde al Tipo III de Gharbi \\
\hline III & $\begin{array}{l}\text { Colección liquida con múltiples septos y } \\
\text { vesículas hijas }\end{array}$ & CE3 & Corresponde al Tipo II de Gharbi \\
\hline IV & Contenido quístico hiperecoico heterogéneo & CE4 & Corresponde al Tipo IV de Gharbi \\
\hline V & $\begin{array}{l}\text { Quiste con pared densa más o menos } \\
\text { calcificada }\end{array}$ & CE5 & Corresponde al Tipo V de Gharbi \\
\hline
\end{tabular}

Fuente: Gharbi HA, Hassine W, Brauner MW, Dupuch K Ultrasound examination of the hydatic liver. Radiology 1981; 139: 459-63.

Mención aparte merece la vómica (eliminación de membranas del quiste por un acceso de tos relatado como expectoración de líquido como clara de huevo y salado) este el síntoma/signo más específico de la afección pulmonar pero que es de presentación poco habitual $(17,10,19)$. Cuando la localización es ósea o muscular los síntomas iniciales son aún más inespecíficos como dolor, masa tumoral, impotencia funcional del miembro o fractura patológica (17).

\section{Estudios por imagen}

La ecografía ha significado un importante avance para el diagnóstico de las hidatidosis hepáticas y de otras localizaciones abdominales asintomáticas siendo la más utilizada en las campañas de tamizaje por ser inocua, de costo accesible, con alta sensibilidad aunque tenga una especificidad menor que la tomografía axial computarizada o la resonancia magnética, además magnética están limitadas a hidatidosis extra abdominal o a pacientes no aptos para la ecografía abdominal, debido a presentar obesidad o meteorismo abundante, quistes complicados para diferenciarlos de los abscesos piógenos o para la planificación de cirugía o radiología intervencionista (23).

La radiografía de tórax muestra un alto rendimiento para el diagnóstico de hidatidosis pulmonar en comparación con los estudios inmunológicos que resultan inespecíficos y con baja sensibilidad debido a que la localización pulmonar estimularía pobremente el sistema inmune (10).

El quiste no complicado aparece como una lesión redondeada homogénea, de bordes bien definidos, tamaño variable, rodeado por pulmón sano o áreas de atelectasia $(24,25)$. 
Sin embargo, a medida que va creciendo puede erosionar los bronquios adyacentes introduciéndose aire entre la membrana periquistica y membrana subsiguiente "signo de doble arco", si a esto se agrega aire dentro del quiste adquiere el aspecto de cascara de cebolla "signo de cumbo" si con la tos y la expectoración se elimina parte del fluido, las membranas colapsan y caen dentro del quiste "signo de la serpiente", luego cuando el endoquiste colapsa totalmente flota en el fluido residual configura el "signo del camalote", finalmente tras la eliminación total del fluido hidatídico los remanentes solidos caen hacia la parte, mas declive de la cavidad constituyen el signo de "masa dentro de la cavidad" $(19,24,25)$.

Lastimosamente en el Perú en todos los trabajos revisados no se utiliza ninguna de estas clasificaciones ni se describen ninguno de los signos pulmonares lo cual dificulta la identificación del estadio de la
Se está dejando de usar la hemaglutinación indirecta (HIA) porque son muy sensibles pero poco específicos así como la inmunoelectroforesis con detección del arco $5^{\circ}$ (DD5) que contrariamente son muy específicos $98 \%$ pero poco sensibles menor al $60 \%$, de este modo, una reacción negativa de ambas pruebas no descarta el diagnóstico (26).

El inmunodiagnóstico es de bastante utilidad frente a imágenes sospechosas o frente al diagnóstico diferencial de una lesión tumoral y debiera solicitarse de rutina, con las limitaciones descritas en las zonas de alta endemicidad como la sierra central del Perú. Las reacciones serológicas se negativizan en uno a cuatro años post cirugía, cuando no existe recidiva (26).

Los exámenes de laboratorio de rutina son inespecíficos, siendo más frecuente la eosinofilia de más de $5 \%$ o de más de 300 células por mm3, signo que es compartido

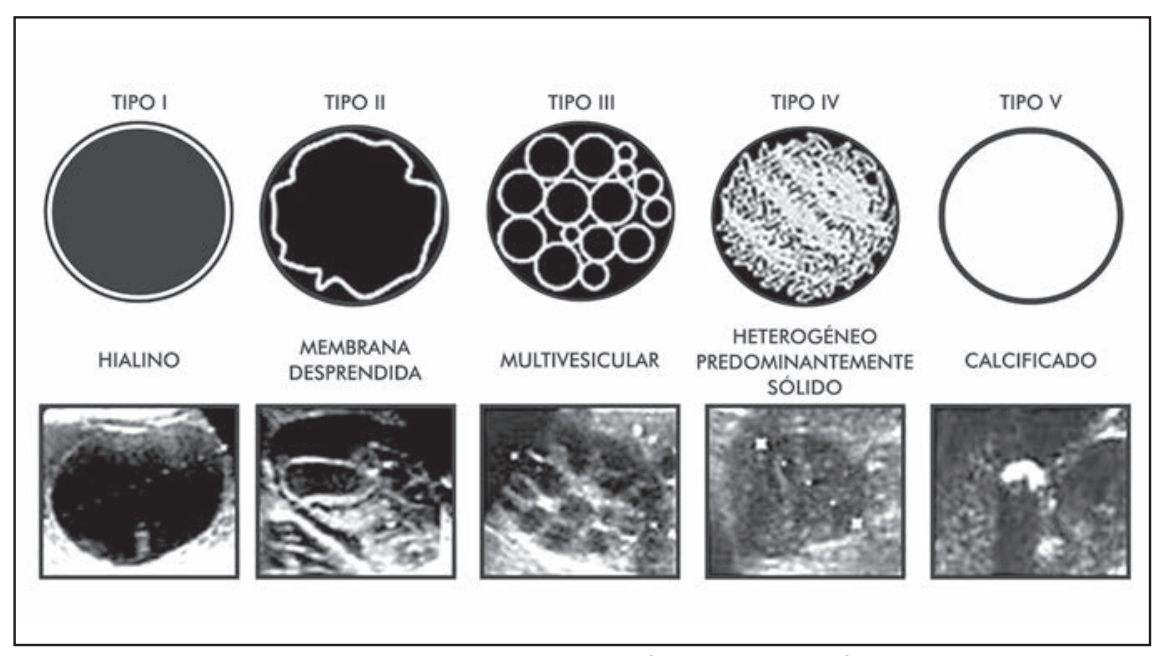

Figura $N^{\circ}$ 3: Comparación de las imágenes ecográficas de las clasificaciones de Gharbi y de la OMS.

Fuente: Electronic Presentation Online System. 2012. Recuperado de: http://posterng.netkey.at/ esr/viewing/index.php? module=viewing_poster\&task=viewsection\&ti $=365193$

hidatidosis y la posterior conducta terapéutica.

\section{Exámenes de laboratorio}

En la actualidad las pruebas diagnósticas más usadas para determinar los antígenos parasitarios son la determinación sérica de inmunoglobulinas G (ELISA$\lg$ ) con una sensibilidad de $86 \%$ y una especificidad de $93 \%$ y el "Western Blot" basado en los mismos principios del ELISA-IgG con una sensibilidad y especificidad del $96 \%(2,26)$ sin embargo es importante destacar que estas pruebas tienen una baja sensibilidad y especificidad en hidatidosis extrahepatica llegando a un 50 y $25 \%$ respectivamente, por lo que la negatividad de estas pruebas no descarta el diagnóstico en estos casos (22). con otras parasitarios como la ascariasis, triquinosis y cisticercosis, también puede encontrarse leucocitosis cuando el quiste presenta alguna complicación de tipo infeccioso (13). Las alteraciones de las pruebas de función hepático (transaminasas, fosfatasa alcalina, bilirrubinas) sugieren complicaciones del quiste $\mathrm{O}$ compromiso de la vía biliar (rotura, abscedación) (13).

\section{Anatomía patológica}

El diagnóstico de certeza se logra con la visualización del escólex, directamente o con el estudio anatomopatológico de las dos membranas que presenta el quiste hidatídico, la externa o cutícula y la interna germinativa o prolígera (23). 
La cutícula, es una lámina delgada de $12 \mu \mathrm{m}$, blanquecina, opaca, elástica pero muy frágil, su composición química es semejante a la quitina y es PAS positiva, la membrana germinativa es una capa delgada de15 a $20 \mu \mathrm{m}$ de espesor, de color amarillento y de aspecto finamente granular tiene un aspecto sincisial con numerosos núcleos, muy rica en glucógeno de ella nacen, se nutren y se liberan los escólex (13).

El quiste hidatídico contiene un líquido transparente rico en cloruro de sodio, urea, ácido úrico, vestigios de albúminas y grasas que posee propiedades antigénicas así como elementos macroscópicos (vesículas hijas) como microscópicos (vesículas prolígeras, escólices y ganchitos) (13).

En el $50 \%$ de los casos se ha podido observar una mayor asociación entre fertilidad y quiste pulmonar así como la ausencia de la calcificación de su adventicia $(7,20)$ distinto al quiste hepático donde la calcificación tardía suele verse hasta en el $10 \%$ de los casos (23).

\section{Tratamiento}

Según la clasificación de la OMS existen cuatro alternativas de tratamiento para la hidatidosis, la cirugía, el PAIR (punción, aspiración, inyección, reaspiración), farmacológico y el "observar y esperar", la mejor opción dependerá de la experiencia del grupo tratante, del número y localización de los quistes, de la existencia de complicaciones asociadas, de la evidencia acumulada en la literatura médica y de las preferencias del paciente $(2,7)$.

La cirugía es el tratamiento de elección en los quistes grandes o infectados o con posibilidad de ruptura y para los quistes localizados en órganos importantes, en general existen 2 tipos de cirugía, la radical que consiste en una periquistectomía y en algunos casos segmentectomía o lobectomía, la conservadora que consiste en el drenaje de la cavidad con una remoción parcial de las membranas, está última está asociada a un mayor número de complicaciones tales como fístula biliar o absceso de la cavidad $(7,21)$.

Se prefiere la cirugía radical frente a la conservadora por su eficacia del $100 \%$ cuando se logra la extirpación completa de la membrana germinativa y no se dejan cavidades residuales ni comunicaciones con el exterior, además tiene una menor recidiva local y menor tasa de morbilidad post operatoria así como de complicaciones hepáticas $(7,21)$.

El mismo principio de cirugía radical o conservadora se utiliza en la hidatidosis pulmonar, la primera consiste en una segmentectomía, lobectomía o neumonectomía y está reservada para pulmones muy afectados, en la cirugía conservadora se realiza una quistostomía (eliminación del parásito o sus restos sin resecar el tejido pulmonar ósea se deja la adventicia) o una quistectomía (resección de todos los componentes del quiste) dependiendo de la técnica que se utiliza $(10,22,23)$.

Para el tratamiento de la cavidad parenquimal que queda luego de la quistectomía se cierran los bronquios abiertos hacia la cavidad (aerostasia) y en cavidades grandes se realiza capitonaje (sutura con puntos que abarcan todo el espesor parietal) $(7,20)$. La quistostomía más capitonaje con cierre de aperturas bronquiales ha demostrado que tiene una alta eficacia terapéutica con menos del $2 \%$ de recurrencia a largo plazo (21).

A diferencia del quiste hepático donde se realiza una resección periquistica ya sea segmentectomía o lobectomía en la cirugía pulmonar no se recomienda remover la zona circundante por el riesgo de desarrollar fístulas broncopleurales, (20) que en las cirugías conservadoras se pueden presentar hasta en el $35 \%$ de los casos $(6,14,20)$.

La PAIR, desarrollada en la década de 1980, comprende la aspiración de los quistes por punción percutánea con guía ecográfica seguida por inyección de un protoscolicida apropiado (como el cloruro de sodio al $20 \%$ o el etanol al $95 \%$ y la re-aspiración del quiste luego de 15-20 minutos. Un riesgo de esta técnica es la anafilaxia por ello la selección del paciente debe ser adecuada está indicada en tipo CE 1 y CE2 $(2,22)$.

El tratamiento con PAIR está indicado en quistes de hasta $6 \mathrm{~cm}$ y el tratamiento farmacológicos en quistes asintomáticos de hasta $10 \mathrm{~cm}(2,22)$.

Este tratamiento no se realiza en el Perú a pesar que por su baja utilización de recursos podría ser el tratamiento de elección en los países latinoamericanos donde esta enfermedad es híperendémica.

Dentro del tratamiento farmacológico el Albendazol a dosis de $10 \mathrm{mg} / \mathrm{kg}$ (usualmente $400 \mathrm{mg}, 2$ veces por día) luego de una comida con algún contenido graso para mejorar su absorción, sería el fármaco de elección en la actualidad en quistes asintomáticos CE 1 o en pacientes sintomáticos (no complicados) que tienen contraindicación para una cirugía $(2,17,20$, 23).

También es usado 30 días previos a la cirugía para inviabilizar al quiste hidatídico y durante los 60 días posteriores a la cirugía conservadora para disminuir la tasa de recurrencia $(17,20,23)$. Aunque un reciente estudio controlado realizado en 6 grandes centros médicos de 5 países concluyo que su eficacia pudo haber sido exagerada ya que menos del $50 \%$ de 
los pacientes muestra la desaparición de sus quistes, 20 a $25 \%$ no muestra variaciones en su tamaño y cerca de 15 a $30 \%$ presenta recurrencias durante el seguimiento.

El Praziquantel a dosis de $50 \mathrm{mg} / \mathrm{kg} /$ dia tiene un efecto 10 veces mayor que el Albendazol y en combinación con el mismo potencia su efecto en caso de derrame del contenido quístico, (17) un estudio refiere que un tratamiento combinado de Albendazol ( $10 \mathrm{mg} / \mathrm{kg} /$ día) y Praziquantel $(25 \mathrm{mg} / \mathrm{kg} /$ día), administrado durante un mes antes de la cirugía genera más inviabilidad de los protoescolices que la monoterapia con Albendazol. (21) Sin embargo, es importante recordar que los niveles plasmáticos de metabolitos de Albendazol (sulfóxido) se incrementan 4,5 veces si es dado al mismo tiempo que el Praziquantel, y esto puede aumentar la tasa de efectos secundarios por lo cual se necesitan más estudios para evaluar la eficacia de este tratamiento combinado (17). La opción del "Observar y esperar" está indicada en quistes de hasta $4 \mathrm{~cm}$ no complicados tipo CE4 y CE5 los cuales deben ser monitoreados mediante imágenes (especialmente por ecografía),cada 6 meses dado que parte de ellos se calcifican con el tiempo y se vuelven completamente inactivos $(2,21)$.

A diferencia de la hidatidosis hepática donde existen estas opciones de tratamiento en la pulmonar está indicado exclusivamente el tratamiento quirúrgico sean estos sintomáticos o no (17).

\section{CONCLUSIONES}

La hidatidosis es una enfermedad producida por el cestodo Echinococcus granulosus de alta prevalencia en el Perú y más aún en la región central andina (Cerro de Pasco, Junín, Huancavelica), tiene un lento crecimiento dentro de los órganos humanos pudiendo ser asintomáticos hasta la edad adulta, los órganos más afectados son el hígado y el pulmón donde son diagnosticados por sus antecedentes epidemiológicos, clínica sugestiva (dolor abdominal, vómica) estudios de imagen principalmente ecografía abdominal, radiografía de tórax y exámenes de laboratorio como el Elisa lgG y Wester Blot.

Dependiendo del tamaño la OMS plantea 4 opciones de tratamiento, la cirugía el PAIR (punción, aspiración, inyección, re-aspiración), el farmacológico y el "observar y esperar". En el Perú, por desconocimiento y falta de insumos se emplea principalmente el tratamiento quirúrgico. Este estudio se hizo para tratar de estandarizar el diagnóstico y manejo de esta enfermedad a través de guías con evidencia científica aceptada en la literatura médica.

\section{REFERENCIAS BIBLIOGRÁFICAS}

1. Craig PS, McManus DP, Lightowlers MW, et al. Prevention and control of cystic echinococcosis. Emerg Infect Dis. 2008; 14(2): 260-266.

2. McManus DP, Gray DJ, Zhang W, Yang Y. Diagnosis, treatment, and management of echinococcosis. BMJ. 2012; 344: 3866-3870.

3. Moro P, Schantz P. Echinococcosis: a review. Int J Infect Dis. 2009; 13(2): 125-133.

4. Irabedra P, Salvatella R. El proyecto subregional cono sur de control y vigilancia de la hidatidosis. Rev Peru Med Exp Salud Pública. 2010; 27(4): 598-603.

5. Moro PL, Lopera L, Cabrera M, Cabrera G, Silva B, Gilman RH, Moro MH. Short report: endemic focus of cystic echinococcosis in a coastal city of Peru. Am J Trop Med Hyg. 2004; 71 (3): 327-329.

6. Salgado S, Suarez L, Cabrera R. Características clínicas y epidemiológicas de la equinococosis quística registrados en un área endémica en los andes centrales del Perú. Neotropical Helminthology. 2007; 1(2): 69-83.

7. Fica A, Soto A, Slater J, et al. Quince años de experiencia clínica con hidatidosis. Revista chilena de infectología. 2012; 29(2): 183-191.

8. Moldovan R, Neghina AM, Calma CL, Marincu I, Neghina R. Human cystic echinococcosis in two south-western and central-western Romanian counties: a 7-year epidemiological and clinical overview. Acta Trop. 2012; 121(1): 26-29.

9. Djuricic SM, Grebeldinger S, Kafka DI, Dian I, Vukadin M, Vasiljevic ZV. Cystic echinococcosis in children - the seventeen-year experience of two large medical centers in Serbia. Parasitol Int. 2010; 59(2): 257-261.

10. Rafael A, Ramos W, Peralta J, Rojas L, Montesinos E, Ortega-Loayza A. Hidatidosis pulmonar en un hospital de Lima, Perú: experiencia en 113 pacientes. Rev. Perú. Med. Exp. Salud Pública. 2008; 25(3): 285-289.

11. Lahiri S. Physiological response and adaptations to high altitude. International Review of hysiology. 1977; 15: 217-251.

12. Moro PL, McDonald J, Gilman RH, Silva B, Verastegui $M$, Malqui $V$, Lescano $G$, Falcon N, Montes G, Bazalar H. Epidemiology of Echinococcus granulosus infection in the central Peruvian Andes. Bull World Health Organ 1997; 75: 553-561.

13. Vera MG, Venturelli MF, Ramírez TJ, et al. Hidatidosis humana. Artículo de actualización, Cuad. Cir. 2003; 17: 88-94.

14. García P, Chabes A, Fernández M.et al. Características clínicas y epidemiológicas de hidatidosis en el Hospital Nacional Dos de Mayo. Revista de Medicina Humana Universidad Ricardo Palma. 2006; 6 (2): 26-30. 
15. Anzieta VJ, Caro DM, Fierro AC. Rocco RE. Quiste hidatídico pulmonar en niños Cuad. Cir. 2002; 16: 16-19.

16. Martinez P. Caracterización de la mortalidad por hidatidosis humana Chile 2000-2010. Rev Chilena Infectol. 2014; 31(1): 7-15.

17. Moral M, Laplume H, Camera L, et al. Enfermedades infecciosas. Hidatidosis, Guía para el equipo de salud. Buenos Aires: Dirección de Epidemiologia/Ministerio de Salud de la Nación Argentina; 2012. Disponible en: http://www.msal. gov.ar/images/stories/epidemiologia/pdf/guiamedica-hidatidosis.pdf

18. Gómez O, Sánchez ED, Jozami D, et al. Hidatidosis, enfermedad de denuncia obligatoria. Revista Medicina de Buenos Aires. 2006; 166(1): 7-8.

19. Bencomo O, Cuesta O, Rubio JM, et al. Hidatidosis pulmonar en pacientes bajo tratamiento sustitutivo de la función renal. Rev Ciencias Médicas. 2011; 15(2): 303-310.

20. Manterola C, Cuadra A, Fonseca F, et al. Utilidad de DD5 y Elisa-IGg como pruebas diagnósticas específicas en pacientes con hidatidosis hepática. Rev. Chilena de Cirugía. 2003; 55(1): 25-29.

21. Cubas R, Brain C, López-Guillemain R. Hidatidosis hepática. Experiencia en 25 años. Cir. 2011; 79: 331-337.

22. Pinto P. Actualización en el diagnóstico y tratamiento de la hidatidosis hepática. Rev. Chilena de Cirugía. 2008; 60(6): 561-566.

23. Hosch W, Junghanss T, Werner J, Düx M. Imaging methods in the diagnosis and therapy of cystic echinococcosis. Rofo. 2004; 176(5): 679-687.

24. Larrieu E, Frider B, Del Carpio M, et al. Portadores asintomáticos de hidatidosis epidemiologia diagnostico y tratamiento. Revista Panamericana de Salud Pública. 2000; 8(4): 250-256.

25. Oviedo I, Castillo E. Diagnóstico por imagen de un quiste hidatídico pulmonar gigante. Reporte de un caso clínico. Gac Med Bol. 2013; 36(1): 3941.

26. Muños P. Diagnóstico y tratamiento de la hidatidosis. Rev Chil Infect. 2007; 24(2): 153-154. 